\title{
Recurrent Intestinal Obstruction Caused by Abdominal Cocoon
}

\author{
Rohit Bansal, D. P. Singh \\ Department of Surgery, Government Medical College \& Rajindra Hospital, Patiala, India \\ Email: rohitbansal541@gmail.com
}

Received 31 May 2014; revised 25 June 2014; accepted 17 July 2014

Copyright (C) 2014 by authors and Scientific Research Publishing Inc.

This work is licensed under the Creative Commons Attribution International License (CC BY). http://creativecommons.org/licenses/by/4.0/

(c) (i) Open Access

\begin{abstract}
Abdominal cocoon is a rare disease of the peritoneum and the rare cause of intestinal obstruction. The etiology of this disease is largely unknown. It primarily affects young adolescent females. We report a case of abdominal cocoon in a 16-year-old girl patient who presented with recurrent episodes of subacute intestinal obstruction.
\end{abstract}

\section{Keywords}

\section{Abdominal Cocoon, Unknown Etiology, Intraoperative Diagnosis}

\section{Introduction}

Intestinal obstruction is a commonly encountered surgical emergency, and usually occurs secondary to intestinal adhesions, cancer, obstructed hernia, etc. We reported a case of a 16-year-old young female who was admitted in our ward with c/o pain abdomen and obstipation for two times for last 6 months. Every time diagnosis of subacute intestinal obstruction was made which was relieved by conservative management. This was the third time that she presented again with subacute intestinal obstruction and laparotomy was done which showed abdominal cocoon as the cause of recurrent intestinal obstruction.

\section{Case Report}

A 16-year-old girl was admitted in emergency ward complaining of pain whole abdomen and obstipation for 2 - 3 days. On examination her abdomen was soft nontender with mild distension, hernia sites were also normal. Vitals were within normal limits. X-ray abdomen erect and supine were s/o multiple air fluid levels and ultrasound abdomen showed no free fluid. There was no history of any abdominal surgery, no history of weight loss, anorexia, menstrual cycles were also normal. Diagnosis of subacute intestinal obstruction was made with no diagnostic clue about cause of intestinal obstruction and patient was managed conservatively and discharged home. She was ad- 
mitted third time again in our ward with similar complaints and now the decision for exploratorylaparotomy was made. On surgery, a fibrous capsule covering all the abdominal viscera was revealed, in which small bowel loops were encased, with the presence of interloop adhesions (Figure 1). Incision of the thick membrane and extensive adhesiolysis of small bowel loops was performed (Figure 2) without loop resection and ileostomy was done. Biopsy of membrane was taken which was suggestive of thick collagenous granulomatous lesion. Patient was put on antitubercular treatment for nine months keeping in mind possibility of tubercular etiology and after that ileostomy closure was done and patient made uneventful recovery with more than 8 months follow up. A diagnosis of idiopathic sclerosing encapsulating peritonitis (abdominal cocoon) was established, due to intraoperative findings and by ruling-out any other specific provenedcondition explaining the patient's pathology.

\section{Discussion}

The abdominal cocoon remains an uncommon cause of intestinal obstruction, and a search of Medline revealed only about 60 articles dealing with this topic till date. Of these, the majority of cases reported were of the primary type, but the secondary form was also frequently reported, although we could come across only 3 cases

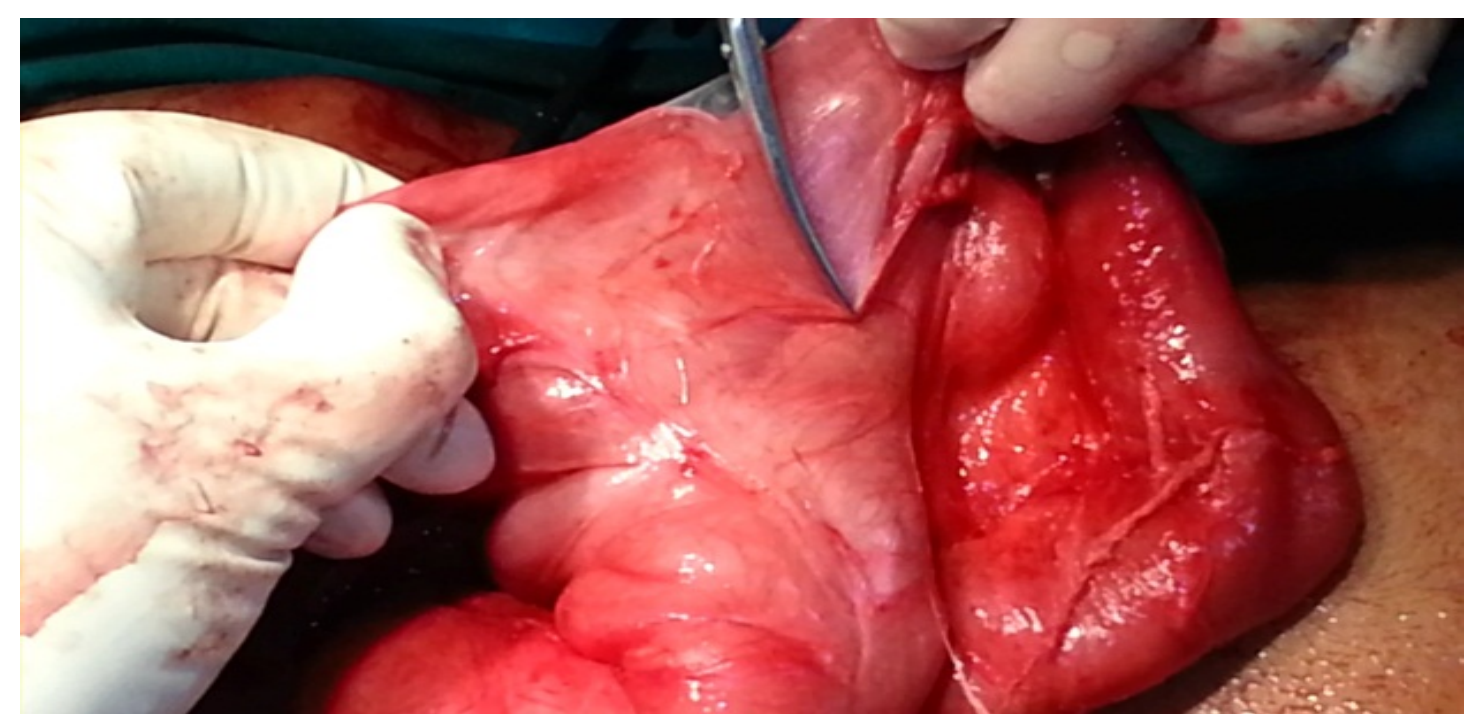

Figure 1. Showing fibrous capsule encasing small bowel loops.

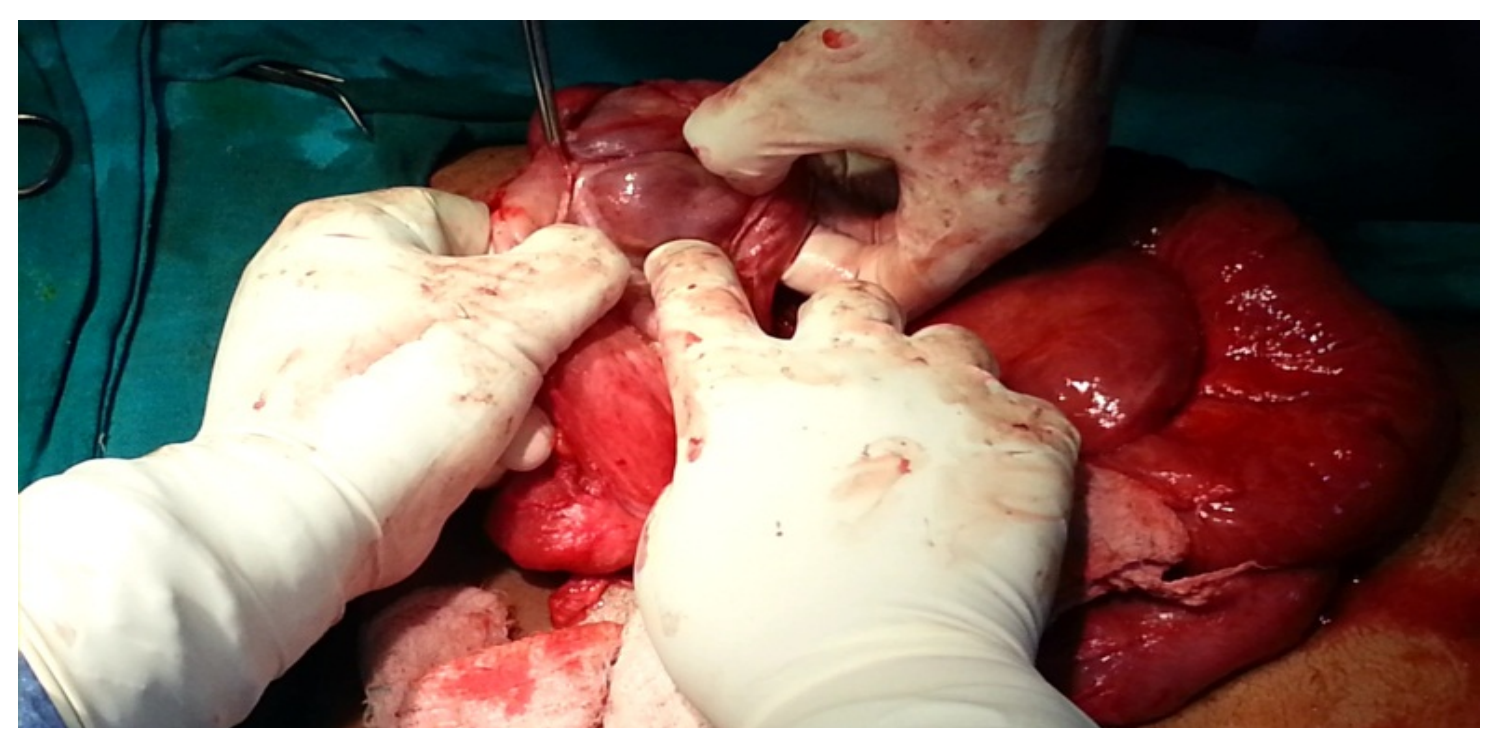

Figure 2. Showing adhesiolysis with small bowel loop visible. 
with a possible tubercular aetiology—one from the original report by Foo et al. [1] another in the report by Sahoo et al. [2] and the third, in the report of Lalloo et al. [3]. Clinically, these patients with abdominal cocoon present with attacks of colicky pain abdomen, nausea, vomiting with intestinal obstruction that is seldom complete. An abdominal mass may or may not be present [4]. Although some authors have described a few radiological signs on plain X-ray, barium series and computerized tomogram scan, it is, as a rule, difficult to be able to make a definite pre-operative diagnosis of this entity [5] [6]. The diagnosis is usually made at laparotomy, when the encasement of the small bowel within the sac-like cocoon is visualized. Although the disease primarily involves small bowel, it can extend to involve other organs like the large intestine, liver and stomach. The treatment is by lysis of this covering membrane, and rarely, further procedures such as resection, are required [7].

\section{Conclusion}

In the case presented, an intra-operative diagnosis of idiopathic sclerosing peritonitis was made in a young girl patient with a history of recurrent bouts of intestinal obstruction. Pre-operative findings were inconclusive. Biopsy was suggestive of chronic granulomatous lesion possibility of tuberculosis. Patient was put on antitubercular treatment. After 9 months of treatment ileostomy closure was done and patient made an uneventful recovery.

\section{References}

[1] Foo, K.T., Ng, K.C., Rauff, A., Foong, W.C. and Sinniah, R. (1978) Unusual Small Intestinal Obstruction in Adolescent Girls: The Abdominal Cocoon. British Journal of Surgery, 65, 427-430. http://dx.doi.org/10.1002/bjs.1800650617

[2] Sahoo, S.P., Gangopadhyay, A.N., Gupta, D.K., Gopal, S.C., Sharma, S.P. and Dash, R.N. (1996) Abdominal Cocoon in Children: A Report of Four Cases. Journal of Pediatric Surgery, 31, 987-988. http://dx.doi.org/10.1016/S0022-3468(96)90431-5

[3] Lalloo, S., Krishna, D. and Maharajh, J. (2002) Abdominal Cocoon Associated with Tuberculous Pelvic Inflammatory Disease. British Journal of Radiology, 75, 174-176. http://dx.doi.org/10.1259/bjr.75.890.750174

[4] Kittur, D.S., Korpe, S.W., Raytch, R.E. and Smith, G.W. (1990) Surgical Aspects of Sclerosing Encapsulating Peritonitis. Archives of Surgery, 125, 1626-1628. http://dx.doi.org/10.1001/archsurg.1990.01410240108022

[5] Hamaloglu, E., Altun, H., Ozdemir, A. and Ozenc, A. (2002) The Abdominal Cocoon: A Case Report. Digestive Surgery, 19, 422-424. http://dx.doi.org/10.1159/000065827

[6] Deeb, L.S., Mourad, F.H., El-Zein, Y.R. and Uthman, S.M. (1998) Abdominal Cocoon in a Man. Preoperative Diagnosis and Literature Review. Journal of Clinical Gastroenterology, 26, 148-150. http://dx.doi.org/10.1097/00004836-199803000-00013

[7] Seng, L.K., Mahadevan, M. and Musa, A. (1993) Abdominal Cocoon: A Report of Two Cases. British Journal of Surgery, 80, 1149. http://dx.doi.org/10.1002/bjs.1800800928 
Scientific Research Publishing (SCIRP) is one of the largest Open Access journal publishers. It is currently publishing more than 200 open access, online, peer-reviewed journals covering a wide range of academic disciplines. SCIRP serves the worldwide academic communities and contributes to the progress and application of science with its publication.

Other selected journals from SCIRP are listed as below. Submit your manuscript to us via either submit@scirp.org or Online Submission Portal.
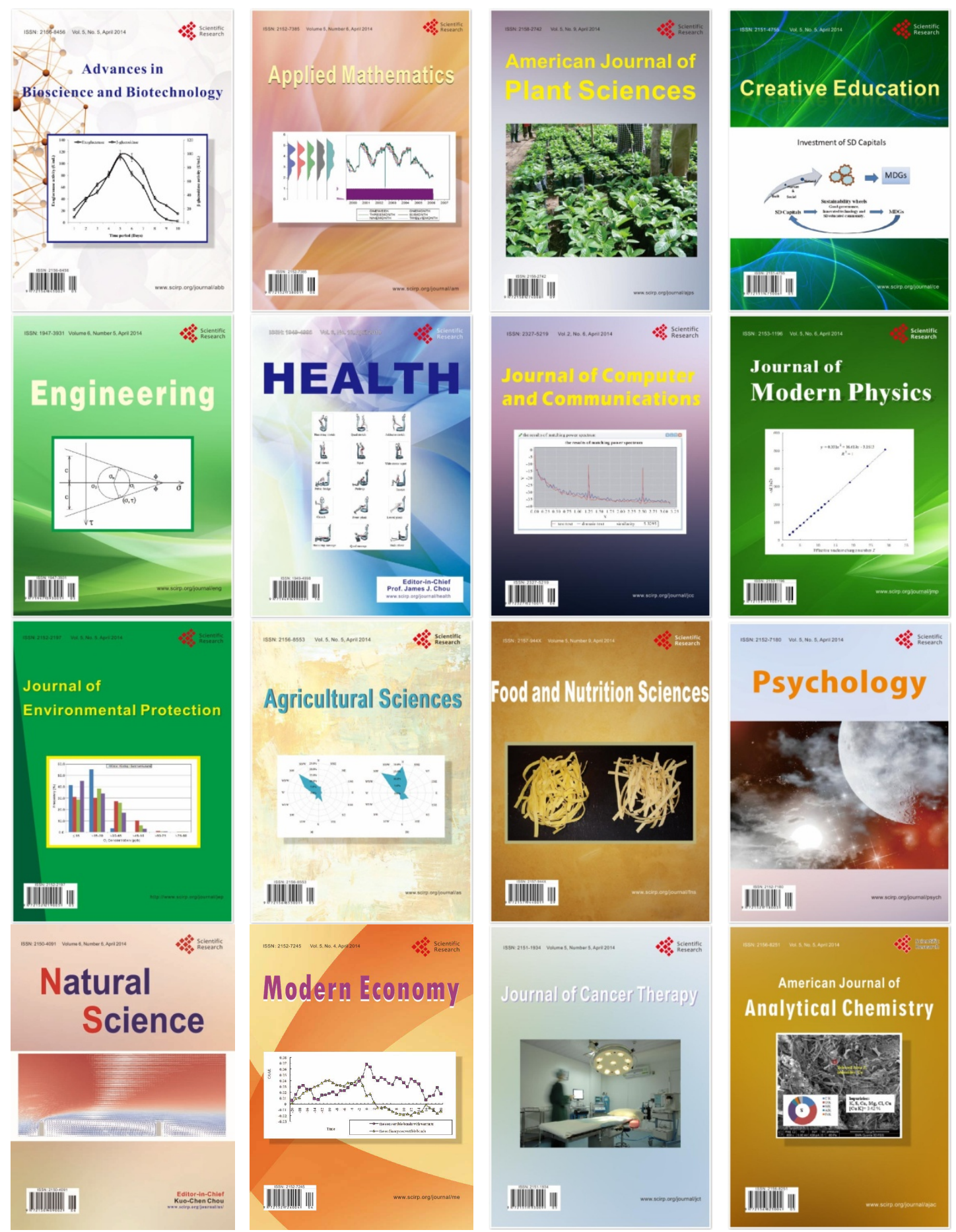